\title{
ERRATUM
}

Open Access

\section{Erratum to: Ethnoichthyology of the indigenous Truká people, Northeast Brazil}

Carlos Alberto Batista Santos ${ }^{1,2^{*}}$ and Rômulo Romeu Nóbrega Alves ${ }^{3}$

\section{Erratum}

The original version of this article [1] unfortunately contained a mistake. In the author list, the surname of author Rômulo Romeu Nóbrega Alves was incorrectly spelled. The original article has now been updated with the correct spelling.

\section{Author details}

'Programa de Pós Graduação em Etnobiologia e Conservação da Natureza, Departamento de Ciências Biológicas, Universidade Federal Rural de Pernambuco, Rua Dom Manoel de Medeiros, s/n, Dois Irmãos, 52171-900 Recife, PE, Brazil. ${ }^{2}$ Departamento de Tecnologia e Ciências Sociais, Universidade do Estado da Bahia, Avenida Edgard Chastinet, s/n, São Geraldo, 48905-680 Juazeiro, BA, Brazil. ${ }^{3}$ Departamento de Biologia, Universidade Estadual da Paraíba, Av. das Baraúnas, 351/Campus Universitário, Bodocongó, 58109-753 Campina Grande, Paraíba, Brazil.

Published online: 14 January 2016

\section{References}

1. Santos CA, Nóbrega Alves RR. Ethnoichthyology of the indigenous Truká people, Northeast Brazil. J Ethnobiol Ethnomed. 2016;12:1. doi:10.1186/ s13002-015-0076-5.

\footnotetext{
* Correspondence: cacobatista@yahoo.com.br

'Programa de Pós Graduação em Etnobiologia e Conservação da Natureza, Departamento de Ciências Biológicas, Universidade Federal Rural de Pernambuco, Rua Dom Manoel de Medeiros, s/n, Dois Irmãos, 52171-900 Recife, PE, Brazil

${ }^{2}$ Departamento de Tecnologia e Ciências Sociais, Universidade do Estado da Bahia, Avenida Edgard Chastinet, s/n, São Geraldo, 48905-680 Juazeiro, BA, Brazil

Full list of author information is available at the end of the article
}

\section{Submit your next manuscript to BioMed Central and take full advantage of:}

- Convenient online submission

- Thorough peer review

- No space constraints or color figure charges

- Immediate publication on acceptance

- Inclusion in PubMed, CAS, Scopus and Google Scholar

- Research which is freely available for redistribution

Submit your manuscript at

www.biomedcentral.com/submit

() BioMed Central 